\title{
A special issue on structural proof theory, automated reasoning and computation in celebration of Dale Miller's 60th birthday
}

\author{
David Baelde, Amy Felty, Gopalan Nadathur and Alexis Saurin
}

The genesis of this special issue was in a meeting that took place at Université Paris Diderot on December 15 and 16, 2016. Dale Miller, Professor at École polytechnique, had turned 60 a few days earlier. In a career spanning over three decades and in work conducted in collaboration with several students and colleagues, Dale had had a significant influence in an area that can be described as structural proof theory and its application to computation and reasoning. In recognition of this fact, several of his collaborators thought it appropriate to celebrate the occasion by organizing a symposium on topics broadly connected to his areas of interest and achievements. The meeting was a success in several senses: it was attended by over 35 people, there were 15 technical presentations describing new results, and, quite gratifyingly, we managed to spring the event as a complete surprise to Dale.

The quality of the presentations at the event and the appropriateness at that time of taking stock of the research accomplishments in the area prompted the consideration of a publication dedicated to the occasion. This special issue is, in a sense, a realization of that thought. The novelty of the ideas that had been presented at the meeting made a journal such as Mathematical Structures in Computer Science (MSCS) a natural choice for a publication vehicle. After obtaining an endorsement from MSCS, a call-for-papers was circulated to a variety of mailing lists. This call circumscribed the themes to be broadly ones to which Dale had made pioneering contributions, so that the special issue could legitimately be referred to as a Miller Festschrift. Topics indicated to be relevant in the call encompassed at least those of structural proof theory, proof search and computation, foundations of logic programming, higher-order unification, reasoning about relational specifications, and certification of proofs. Quite importantly, submission was open to all, i.e., it was not limited to the participants at the event in Paris, and acceptance was to be based on meeting normal journalistic standards. Sixteen manuscripts were received in response to the call and these were all subjected to rigorous review: almost all the manuscripts received three independent reviews and several of them went through multiple stages of revision. The 12 papers in this special issue are the ones that were accepted at the end of the process.

The papers in the issue have been grouped together based on the themes they cover and these groups have then been arranged in roughly the chronological order in which Dale's research has touched these topics. The first group, comprising the first three papers, concerns proof formats and the essential content of proofs, matters that are of interest in the context of automated reasoning. Two of these papers build on the notion of expansion trees that Dale introduced in his doctoral thesis as a compact representation for cut-free proofs in classical higher-order logic. The next two papers constitute a group concerned with proof search and with inhabitation questions related to type systems. The second of these papers proposes to augment $\lambda$ Prolog, a language coinvented by Dale, with constraints and to use the resulting language as a vehicle for implementing proof search in pure type systems. The next three papers comprise a group that pertains broadly to structural proof theory, a topic to which Dale has made several noteworthy contributions. These papers variously describe proof systems for extensions of linear logic, study relationships between 
variants of this logic, and examine decidability questions for such variants. The ninth paper in the collection develops a semantical understanding of the nabla quantifier, a logical device introduced by Dale and Alwen Tiu to capture a form of generic quantification. The final three papers constitute a group pertaining to varied aspects of specifying object systems and reasoning about them through such specifications. The last of these papers is especially concerned with the issue of associating with any claimed theorem a proof that can be checked independently by a skeptical consumer. This is the topic of proof certification that has commanded much attention of late and on which Dale has recently led a large research project.

The production of this special issue has been somewhat delayed: we had originally projected that it would have been completed a year closer to the event that it was loosely celebrating. In retrospect, the development of a carefully reviewed collection of papers of this kind is a significant undertaking and we were perhaps overly ambitious in our initial plans. We are grateful to the authors for their willingness to accept the deadlines we set for them and for their indulgence in the face of delays. We are also indebted to the reviewers who, in some cases, have had to read variants of a manuscript two or three times and have still been willing to provide critical appraisals; we believe that their diligence shines through in the quality of the papers in the collection. Finally, to our colleague Dale, we hope that this special issue reflects in a small way the success of your past research endeavors, and that it also provides additional inspiration for many more years of leading and participating in such developments. 